\title{
COMMUNICATION
}

\section{A plasmonic nanosensor with inverse sensitivity for circulating cell-free DNA quantification}

Cite this: DOI: $10.1039 / \times 0 \times x 00000 x$

\author{
Roger M. Pallares, ${ }^{a, b}$ Say Li Kong, ${ }^{\mathrm{c}}$ Tan Hui Ru, ${ }^{\mathrm{b}}$ Nguyễn T.K. Thanh, ${ }^{d}$ Yi Lu ${ }^{\mathrm{e}}$ and \\ Xiaodi $\mathrm{Su}^{*}$
}

Received 00th January 2012,

Accepted 00th January 2012

DOI: $10.1039 / \times 0 \times x 00000 x$

www.rsc.org/

A plasmonic nanosensor (using gold nanorods) with inverse sensitivity is presented for circulating cell-free DNA quantification. The inverse sensitivity (i.e. the lower the analyte concentration, the higher the response intensity) is achieved by the unusual DNA concentration-dependent gold nanorod aggregation. This assay method can adjust the dynamic range by controlling the concentration of nanoparticles in solution.

Currently, biopsy is the only method that can diagnose cancer with absolute certainty. ${ }^{1}$ This medical test involves the removal of tissue from the patient to determine the presence and extent of the abnormal cell growth. Several non-invasive alternatives have been developed, such as body fluid analysis. ${ }^{2-4}$ However, the lack of sensitivity and specificity of the most serum cancer biomarkers has prevented the use of body fluid analysis as definitive non-invasive sensing technique for cancer diagnostics. ${ }^{5,6}$ Nevertheless, the analysis of circulating cell-free DNA (cfDNA), i.e. extracellular and mostly double-stranded DNA found in nucleosomes, ${ }^{7,8}$ and other complex species ${ }^{8-10}$ in blood, serum and plasma, has recently emerged as a promising new non-invasive liquid biopsy, which allows monitoring the patient's therapeutic response and disease progression. ${ }^{11-13}$ Even though a few commercial kits are available, providing fast and easy-to-use DNA quantification, most of them are not able to cover all the physiological cfDNA concentration range. Furthermore, the ranges of concentrations of the analysed samples are frequently close to the limit of detection (LOD) of those kits, providing small intensity responses, that leads to a low reliability. Therefore, new assay concepts are required for a robust quantification of cfDNA at low concentration range, especially around the LOD. In analytical chemistry, the low reliability for low analyte concentrations near the LOD is a common problem. Thus signal amplification strategies, i.e. enzymatic amplification, ${ }^{14}$ labelling the analyte with antibody conjugates ${ }^{15}$ or employing more sophisticate equipment, ${ }^{16}$ have been largely developed. However, those options also increase the complexity of the design and resource investment.

In this work we propose an alternative analytical concept that overcomes the limitations of the commercial kits without involving complex designs. Particularly, we demonstrate a plasmonic nanosensor for cfDNA (or dsDNA) with inverse sensitivity, i.e. the lower the concentration of the analyte is, the higher the response intensity ${ }^{17} \quad$ (Fig. S1). This concept employs hexadecyltrimethylammonium bromide (CTAB) coated gold nanorods (AuNRs) and their electrostatic interactions with dsDNA. The inverse sensitivity is achieved by the unusual DNA concentration-dependent AuNR aggregation, which can be measured by UV-Vis spectroscopy. This sensor is fast $(10 \mathrm{~min})$, straightforward and easy-to-use (one-step, mixture of 3 solutions). To the best of our knowledge, this is the second paper reporting the concept of inverse sensitivity that enables a higher reliability for low concentration analyte detection by creating inverse relationship between analyte concentration and signal output, which introduces high signal-to-noise ratio (SNR) for low concentration detection. The first report of such concept was performed by L. RodriguezLorenzo et al., ${ }^{17}$ where enzymatic catalysed gold nanoparticle formation provides the inverse sensitivity for protein biomarker detection. In our current work, we further reinforce the inverse sensitivity concept for cfDNA detection. Despite of using plasmonic nanoparticles' optical property as a signal output similarly, our method does not involve enzymes and is conceptually simpler without involving long experimental times and multi-step procedures. Furthermore, our concept allows for a tuneable dynamic range not existing in the first inverse sensitivity sensor.

The AuNRs used in this work were synthesized by seedmediated method with $\mathrm{CTAB}$ as a surfactant, ${ }^{18}$ which results in a positively charged gold surface. When negatively charged molecules are mixed with AuNRs, the rods aggregate. ${ }^{19}$ Several reports have published apparently contradictory results, i.e. a few groups reporting the AuNR aggregation by dsDNA ${ }^{20,21}$ while others found that dsDNA can protect them against aggregation. ${ }^{22,23}$ We discover that the result of the interaction between dsDNA and AuNR is concentration dependent, and therefore both induction of AuNR aggregation and protection against aggregation occur depending on the dsDNA and AuNR concentrations. Both phenomena can be followed by the shift of the longitudinal localised surface plasmon resonance band (L-LSPR) as a consequence of the plasmon coupling between contiguous rods.

Fig. 1A shows the UV-Vis spectra of AuNRs (optical density at $890 \mathrm{~nm}, \mathrm{OD}_{890}$ of 0.48 ) with aspect ratio (AR) of 4.9 mixed with dsDNA (in $8 \mathrm{mM}$ Tris buffer) at different concentrations. The 
dsDNA molecules were 180 base-pair long, which is the typical length of the cfDNA fragments originated from apoptotic cells. ${ }^{24}$ The Tris buffer is the most common buffer used by the commercial DNA extraction kits. ${ }^{25}$ Since cfDNA is commonly adsorbed on other species in biological samples (e.g. proteins ${ }^{7,8}$ and lipid membranes ${ }^{8-}$ $\left.{ }^{10}\right)$, its extraction is an essential step prior to the analysis using any commercial quantification kit, ${ }^{26}$ and our plasmonic sensing method. Initially, the dsDNA induces the assembly of the rods, red-shifting the L-LSPR band from 890 to $995 \mathrm{~nm}$ at $2.5 \mathrm{nM}$. However, further addition of dsDNA promotes the opposite phenomenon, i.e. disaggregation of the rods. The L-LSPR band blue-shifts back, up to $895 \mathrm{~nm}$ at $50 \mathrm{nM}$ dsDNA. The aggregation and disaggregation is quantified using the absorbance ratio at 510 and $890 \mathrm{~nm}$ wavelengths (A510/A890) for different dsDNA concentrations in Fig. 1B. The limit of detection (LOD) is $2.5 \mathrm{nM}$, which is calculated as the lowest analyte concentration that is detected in the inverse-sensitivity regime, and the dynamic range is from 2.5 to $50 \mathrm{nM}$. The response curve of the sensor has been divided in two concentration regimes, i.e. below (green) and above (blue) the LOD, respectively. It is noteworthy that the below LOD regime presents normal sensitivity with increasing signal with the analyte concentration. This lower concentration regime is so narrow (5\% of the concentration of the full response curve) that can be neglected, yielding an inverse sensitivity sensor closer to the idealistic performance, i.e. zero response below the LOD. Additionally, the sample concentration within the higher concentration regime (i.e. the one with inverse sensitivity response) can be confirmed by diluting the sample for a second test. An increase of $\mathrm{A}_{510} / \mathrm{A}_{890}$ is expected if the sample is within this higher concentration regime. Otherwise a decrease of $\mathrm{A}_{510} / \mathrm{A}_{890}$ would be observed if it is in the lower concentration regime. Interestingly, the SNR at the LOD is highly enhanced by the inverse sensitivity, e.g. SNR at $2.5 \mathrm{nM}$ is 63 , in comparison to the conventional sensors, whose SNR at the LOD is 3 by definition. ${ }^{27}$
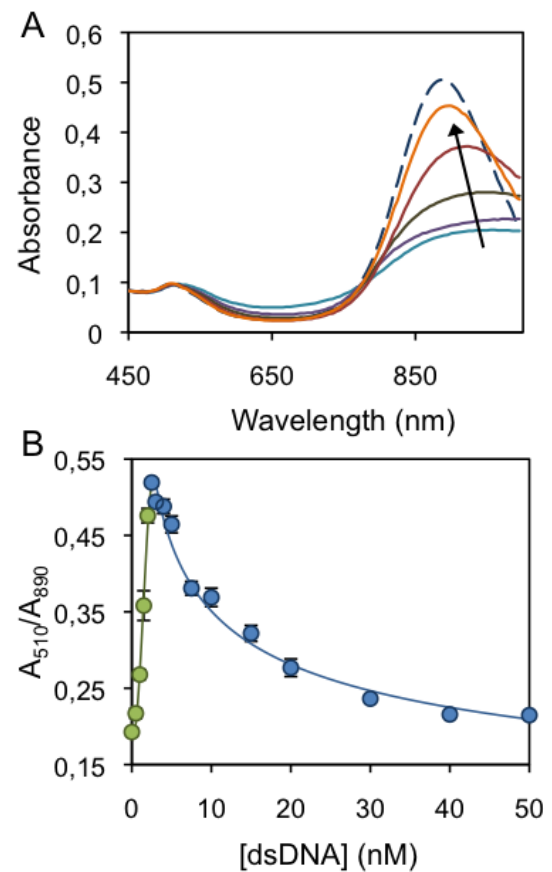

Fig. 1 Characterization of AuNRs $\left(\mathrm{OD}_{890}=0.48\right)$, mixed with different amounts of dsDNA (180bp) in $8 \mathrm{mM}$ Tris buffer. (A) UV-Vis spectra at $0 \mathrm{nM}$ (dashed line), 2, 5, 10, 20 and $40 \mathrm{nM}$ DNA (solid lines). (B) Absorbance ratio intensities at 510 and 890 $\mathrm{nm}$ as the function of DNA concentration. The lower and higher concentration regimes are highlighted in green and blue, respectively.
Transmission electron microscopy (TEM) studies were performed to characterise the AuNRs assembly and disassembly at the nanoscale. Fig. 2A reveals well monodispersed AuNRs in the absence of dsDNA. After the addition of dsDNA of as low as $2 \mathrm{nM}$, the rods are assembled, yielding several micrometer sized aggregates (Fig. 2B). Upon further addition of dsDNA, e.g. $10 \mathrm{nM}$, initiates the disaggregation (Fig. 2C). At dsDNA concentration of $20 \mathrm{nM}$, the original AuNR dispersity is almost recovered and only small aggregates are present in the sample (Fig. 2D). These results are confirmed by dynamic light scattering (DLS), which shows a dramatic increase of the AuNR hydrodynamic radius from 8 to 110 $\mathrm{nm}$, after the initial addition of dsDNA, and its subsequent decrease back to $14 \mathrm{~nm}$ at higher dsDNA concentration (Fig. 2E).

To gain a more complete understanding of the mechanism involved in the concentration-dependent interaction of the dsDNA with the AuNRs, the nanoparticle zeta potential was measured after the addition of different amounts of dsDNA. Fig. 3 shows an initial decrease in the AuNR zeta potential with the increase in the dsDNA concentration. The rapid decrease can be attributed to the screening of the CTAB positive charges by the dsDNA phosphate groups. Once the AuNR net charge has been neutralized, further additions of dsDNA induce a charge reversal and a slow negative increase of the nanoparticle electric potential. Zeta potential is one of the key parameters defining the repulsive forces among nanoparticles and colloidal stability. ${ }^{28}$ Therefore, its fast neutralization and subsequent negative increase resulted in the AuNR initial aggregation and the later disaggregation. This result is consistent with the zeta potential and the $\mathrm{A}_{510} / \mathrm{A}_{890}$ profiles, which show the range of dsDNA concentrations with zeta potential closer to zero is the range with
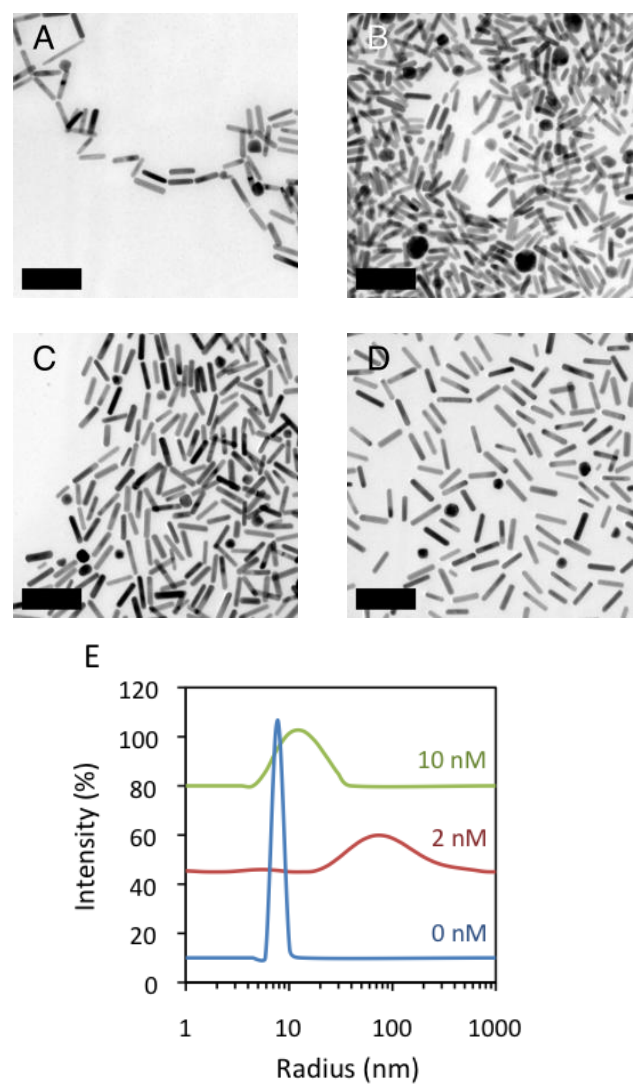

Fig. 2 Characterization of AuNR aggregation and disaggregation induced by dsDNA. TEM images at dsDNA concentrations of (A) 0, (B) 2, (C) 10 and (D) $20 \mathrm{nM}$. (E) Measures of AuNR hydrodynamic radius at 0,2 and $10 \mathrm{nM}$ dsDNA by DLS. The radius distributions have been offset vertically for clarity. 
higher aggregation.

To complement the concentration dependent charge density observations from the zeta potential experiments, we designed an experiment to study the relative positions between AuNRs and dsDNA. In this experiment, the dsDNA molecules were saturated by thiazole orange (TO), i.e. an intercalation dye that increase its fluorescence quantum yield 18900 -fold upon binding to DNA, ${ }^{29}$ at molar ratio of $1: 40$. The fluorescence of the resulting dsDNA-TO can be quenched by AuNRs when they are in close proximity through nanoparticle surface energy transfer mechanism. ${ }^{30}$ Therefore, measuring the fluorescent emission of the dsDNA- $\mathrm{TO}_{40}$ can provide information about their relative position to the AuNR surface. Fig. 4A shows the emission of dsDNA-TO ${ }_{40}$ solutions $(0-50 \mathrm{nM})$, same concentration range as used in the nanorod aggregation study, in the absence and presence of AuNRs $\left(\mathrm{OD}_{890}=0.48\right)$. In the absence of AuNRs, the fluorescence intensity at $535 \mathrm{~nm}$ (maximum emission wavelength) is linearly proportional to the concentration of the dsDNA-TO ${ }_{40}$ complex as expected (Fig. 4B). However, if the measured solution contains AuNRs, the fluorescence is almost totally quenched. Fig. 4C compares the fluorescence emission of dsDNA- $\mathrm{TO}_{40}$ in the presence of AuNRs and the AuNR aggregation profile measured with DNA without the intercalation dye. In the range of concentrations from 0 to $20 \mathrm{nM}$, where the rods are initially aggregated and later start disaggregating, all the fluorescence is quenched. The fluorescence intensity begins increasing after most part of the AuNRs have been disaggregated, e.g. dsDNA-TO ${ }_{40}$ concentrations higher than $20 \mathrm{nM}$.

Based on the collective observations, we propose a mechanism for the DNA concentration-dependent AuNR aggregation and redispersion. Initially, the dsDNA molecules are adsorbed on the AuNR surface by electrostatic interactions, leading to total dsDNA$\mathrm{TO}_{40}$ fluorescence quenching. At the lower DNA concentration regime $(<2.5 \mathrm{nM})$, the electrostatic interactions between the dsDNA molecules and the AuNRs drive the initial aggregation, due to the decrease on the nanoparticle positive charge, and this process continue until the AuNRs have zero net charge. When DNA concentration increases further, more nucleic acids continue to adsorb on the AuNR surface, as evidenced by the nearly total quenching of the dsDNA- $\mathrm{TO}_{40}$ emission up to $\sim 20 \mathrm{nM}$, as well as the pickup of the nanoparticle negative charge. The slow negative charge increase is accountable for the disaggregation process at higher DNA concentration regime $(>2.5 \mathrm{nM})$. At concentrations above $20 \mathrm{nM}$, we hypothesize that the AuNR are mostly covered and the excess dsDNA chains have little access to the CTAB gold surface and thus the fluorescence emission starts to pick up. Those free-DNA molecules have little to non-effect on the nanoparticle disaggregation, based on the small $\mathrm{A}_{510} / \mathrm{A}_{890}$ changes observed at concentrations higher than $20 \mathrm{nM}$. This unique DNA concentration

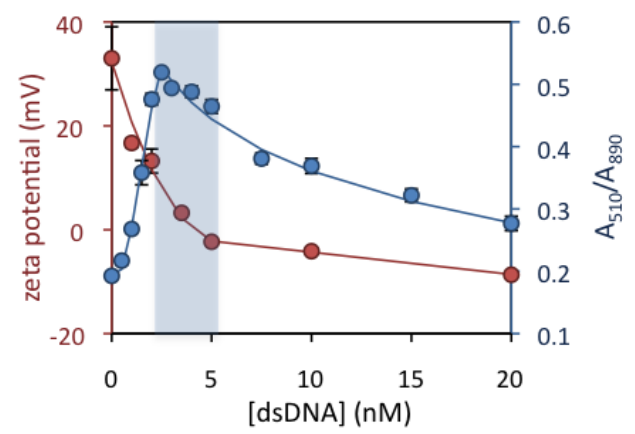

Fig. 3 Comparison between the effect of dsDNA concentration on the AuNR zeta potential (red) and the $\mathrm{A}_{510} / \mathrm{A}_{890}$ (blue). The region of concentrations with higher AuNR aggregation is highlighted in pale blue.
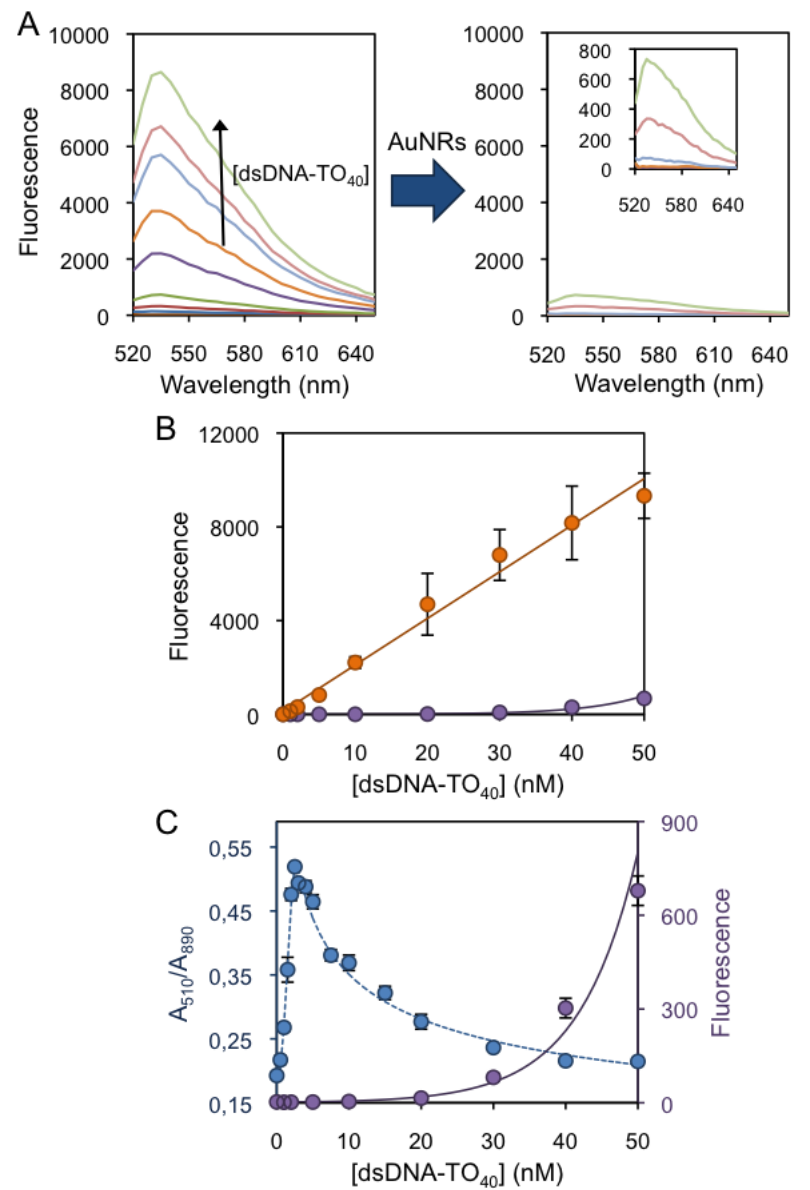

Fig. 4 (A) Fluorescence emission of dsDNA- $\mathrm{TO}_{40}$ solutions in the absence and presence of AuNRs $\left(\mathrm{OD}_{890}=0.48\right)$, respectively. (B) Emission intensities of different dsDNA- $\mathrm{TO}_{40}$ solutions at emission wavelength of $535 \mathrm{~nm}$ in the absence (orange) and presence (purple) of AuNRs. (C) Comparison between the fluorescence emission of dsDNA- $\mathrm{TO}_{40}$ in the presence of AuNR and their aggregation profile. All measures were done with an excitation wavelength of $490 \mathrm{~nm}$.

dependent tuning of AuNR surface charge is the key of the inverse sensitivity.

One major issue for detecting cfDNA and other nuclear acids in clinical samples is that the concentrations vary widely in those samples. To take full advantage of the inverse sensitivity method described here, it is important to tune the dynamic ranges of the detection so that the highest inverse sensitive area matches the cfDNA concentration in the samples. Toward this goal, we have changed the AuNR concentration in solution in order to adjust the dynamic range of the sensor and its section with higher SNR to different common ranges previously published in the literature.

Fig. 5 depicts the aggregation profile of AuNR solutions at five different concentrations, i.e. $\mathrm{OD}_{890}$ of $0.92,0.48,0.22,0.08$ and 0.05 . The LOD was found to increase with the AuNR concentration, e.g. $10 \mathrm{nM}$ for the most concentrated solution, relative to $0.2 \mathrm{nM}$ for the most diluted one. The AuNR concentration also affects the dynamic range, increasing it and shifting it, e.g. from 0.2 to $2 \mathrm{nM}$ and 10 to $100 \mathrm{nM}$ for the AuNR solutions with $\mathrm{OD}_{890}$ of 0.05 and 0.92 , respectively. The combination of the five AuNR solutions yields a sensor that is sensitive enough to monitor the cfDNA levels associated with a wide range of cancer types (Table S2). 


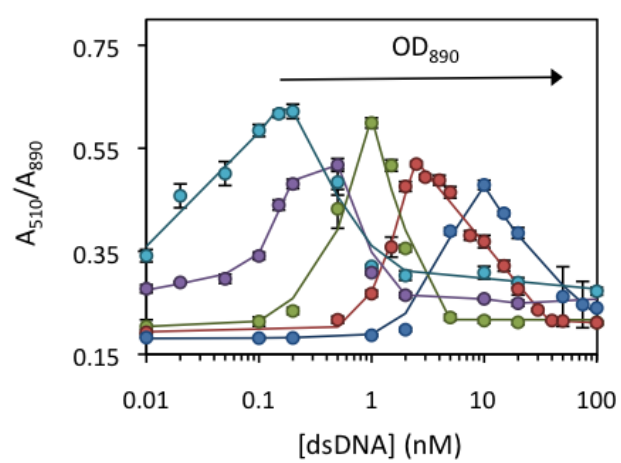

Fig. 5 Effect of the dsDNA concentration on the $\mathrm{A}_{510} / \mathrm{A}_{890}$ of five AuNR solutions. AuNR $\mathrm{OD}_{890}$ of 0.05 (turquoise), 0.08 (purple), 0.22 (green), 0.48 (red) and 0.92 (blue).

In summary, we have developed a plasmonic nanosensor with inverse sensitivity, exploiting the unique DNA concentrationdependent AuNR aggregation/re-dispersion profile for cfDNA detection. A mechanism based on the change of the AuNR electric potential by the adsorption of dsDNA molecules at two regimes of lower and higher concentrations has been proposed to account for the inverse response of the sensor. The LOD and the dynamic range of this method can be adjusted by controlling the AuNR concentration in solution, allowing tunable sensor response curve and covering a wide range of cfDNA concentrations linked to cancer diagnosis and prognosis. The lowest LOD reached by this method is $0.2 \mathrm{nM}$ with an overall dynamic range of 0.2 to $100 \mathrm{nM}$. Notably, this is the second report of inverse sensitivity, relative to a previous one involving enzymatic reaction. The assay is conceptually simple, fast, easy-to-use and compatible with cfDNA extraction medium. This study further reinforces the breakthrough strategy of enhancing the reliability of low concentration detection, by literally introducing high SNR, which is often failed in the normal sensitivity sensing and signal amplification strategies.

\section{Notes and references}

${ }^{a}$ Department of Chemistry, University College London, London, WC1H 0AJ, United Kingdom.

${ }^{b}$ Institute of Materials Research and Engineering, A*STAR (Agency for Science, Technology and Research), 3 Research Link, Singapore, 117602. ${ }^{c}$ Genome Institute of Singapore, A*STAR, 60 Biopolis St, Singapore, 138672

${ }^{d}$ Biophysics Group, Department of Physics and Astronomy, University College London, London, WC1E 6BT and UCL Healthcare Biomagnetic and Nanomaterials Laboratories, 21 Albemarle Street, London W1S 4BS and, United Kingdom.

${ }^{\mathrm{e}}$ Department of Chemistry, University of Illinois at Urbana-Champaign, Illinois, 61801, USA.

Electronic Supplementary Information (ESI) available: See DOI: $10.1039 / \mathrm{c} 000000 \mathrm{x} /$

1 A. Heidenreich, G. Aus, M. Bolla, S. Joniau, V. B. Matveev, H. P. Schmid and F. Zattoni, European Urology, 2008, 53, 68.

2 G. Ploussard and A. de la Taille, Nature Reviews Urology, 2010, 7, 101.

3 S. Hu, J. A. Loo and D. T. Wong, Proteomics, 2006, 6, 6326.

4 E. R. Sauter, W. Zhu, X. J. Fan, R. P. Wassell, I. Chervoneva and G. C. Du Bois, British Journal of Cancer, 2002, 86, 1440.
5 I. M. Thompson, D. K. Pauler, P. J. Goodman, C. M. Tangen, M. S. Lucia, H. L. Parnes, L. M. Minasian, L. G. Ford, S. M. Lippman, E. D. Crawford, J. J. Crowley and C. A. Coltman, New England Journal of Medicine, 2004, 350, 2239.

6 J. R. Prensner, M. A. Rubin, J. T. Wei and Arul M. Chinnaiyan, Science Translational Medicine, 2012, 4, 127rev3.

7 S. Holdenrieder, P. Stieber, L. Y. Chan, S. Geiger, A. Kremer, D. Nagel and Y. D. Lo, Clinical chemistry, 2005, 51, 1544.

8 D. L. Peters and P. J. Pretorius, Clinica Chimica Acta, 2011, 412, 806.

9 P. B. Gahan and M. Stroun, Cell biochemistry and function, 2010, 28, 529.

10 H. D. Halicka, E. Bedner and Z. Darzynkiewicz, Experimental cell research, 2000, 260, 248.

11 V. Swarup and M. R. Rajeswari, FEBS letters, 2007, 581, 795.

12 T. L. Wu, D. Zhang, J. H. Chia, K. C. Tsao, C. F. Sun and J. T. Wu, Clinica Chimica Acta, 2002, 321, 77.

13 A. K. Pathak, M. Bhutani, S. Kumar, A. Mohan and R. Guleria, Clinical Chemistry, 2006, 52, 1833.

14 R. de la Rica and M. M. Stevens, Nature Nanotechnology, 2012, 7, 821.

15 W. C. Law, K. T. Yong, A. Baev and P. N. Prasad, ACS Nano, 2011, 5, 4858.

16 A. Barik, L. M. Otto, D. Yoo, J. Jose, T. W. Johnson and S. H. Oh, Nano Lett., 2014, 14, 2006.

17 L. Rodríguez-Lorenzo, R. de La Rica, R. A. Álvarez-Puebla, L. M. Liz-Marzán and M. M. Stevens, Nature Materials, 2012, 11, 604.

18 B. Nikoobakht and M. A. El-Sayed, Chemistry of Materials, 2003, 15, 1957.

19 G. Kawamura, Y. Yang and M. Nogami, Journal of Physical Chemistry C, 2008, 112, 10632.

20 Z. Maa, L. Tiana, T. Wangb and C. Wangb, Analytica Chimica Acta, 2010, 673, 179.

21 X. J. Zheng, J. D. Qiu, L. Zhang, Z. X. Wang and R. P. Liang, Chem. Commun., 2013, 49, 3546.

22 R. Kanjanawarut and X. Su, Biointerphases, 2010, 5, 98.

23 R. Kanjanawarut, B. Yuan and X. Su, Nucleic acid therapeutics, 2013, 23, 273.

24 S. Jahr, H. Hentze, S. Englisch, D. Hardt, F. O. Fackelmayer, R. D. Hesch and R. Knippers, Cancer Research, 2001, 61, 1659.

25 QIAamp DNA Mini and Blood Mini Handbook, Qiagen, 3rd edn., 2012.

26 T. E. Skvortsova, E. Y. Rykova, S. N. Tamkovich, O. E. Bryzgunova, A. V. Starikov, N. P. Kuznetsova, V. V. Vlassov and P. P. Laktionov, British Journal of Cancer, 2006, 94, 1492.

27 G. L. Long and J. D. Winefordner, Analytical Chemistry, 1983, 55, 712A.

28 D. L. Liao, G. S. Wu, and B. Q. Liao, Colloids and Surfaces A: Physicochemical and Engineering Aspects, 2009, 348, 270.

29 J. Nygren, N. Svanvik and M. Kubista, Biopolymers, 1998, 46, 39.

30 X. Li, J. Qian, L. Jiang and S. He, Applied Physics Letters, 2009, 94, 063111. 